\title{
Correction to: Ethical considerations in conducting surgical research in severe complicated intra-abdominal sepsis
}

Christopher J. Doig ${ }^{1,2}$, Stacey A. Page ${ }^{2}$, Jessica L. McKee ${ }^{3}$, Ernest E. Moore ${ }^{4}$, Fikri M. Abu-Zidan ${ }^{5}$, Rosemary Carroll , John C. Marshall ${ }^{7}$, Peter D. Faris ${ }^{8}$, Matti Tolonen ${ }^{9}$, Fausto Catena ${ }^{10}$, Federico Coccolini ${ }^{11}$, Massimo Sartelli ${ }^{12}$, Luca Ansaloni $^{13}$, Sam F. Minor ${ }^{14}$, Bruno M. Peirera ${ }^{15}$, Jose J. Diaz ${ }^{16}$, Andrew W. Kirkpatrick ${ }^{17,18,19^{*}}$ and Closed Or Open after Laparotomy (COOL) after Source Control for Severe Complicated Intra-Abdominal Sepsis Investigators

\section{Correction to: World J Emerg Surg (2019) 14:39 \\ https://doi.org/10.1186/s13017-019-0259-9}

The original article [1] contained a typo in author, Federico Coccolini's name. This has now been corrected.

\begin{abstract}
Author details
'Department of Critical Care Medicine, Cumming School of Medicine, University of Calgary, Calgary, Canada. ${ }^{2}$ Department of Community Health Sciences, Cumming School of Medicine, University of Calgary, Calgary, Canada. ${ }^{3}$ Regional Trauma Services, Foothills Medical Centre, Calgary, Canada. ${ }^{4}$ University of Colorado, Denver, CO, USA. ${ }^{5}$ Research Facilitation Analytics (DIMR), University of Calgary, Calgary, Alberta, Canada. ${ }^{6}$ Li Ka Shing Knowledge Institute, St. Michael's Hospital, University of Toronto, Toronto, Canada. 'Department of Surgery, College of Medicine and Health Sciences, UAE University, Al-Ain, UAE. ${ }^{8}$ Surgical Services John Hunter Hospital, Newcastle, NSW, Australia. ${ }^{9}$ Department of Abdominal Surgery, Abdominal Center, University of Helsinki and Helsinki University Central Hospital, Helsinki, Finland. ${ }^{10}$ Emergency Surgery Department, Parma University Hospital, Parma, Italy. "'General, Emergency and Trauma Surgery dept, Bufalini Hospital, Cesena, Italy. ${ }^{12}$ Department of Surgery, Macerata Hospital, Macerata, Italy. ${ }^{13}$ Unit of General and Emergency Surgery, Bufalini Hospital of Cesena, Cesena, Italy. ${ }^{14}$ Department of Critical Care and Department of Surgery, NSHA- Queen Elizabeth II Health Sciences Centre, 1276 South Park Street, Halifax, Nova Scotia B3H 2Y9, Canada. ${ }^{15}$ Division of Trauma Surgery, University of Campinas, Campinas, SP, Brazil. ${ }^{16}$ Department of Surgery, Acute Care Surgery, R Adams Cowley Shock Trauma Center, University of Maryland School on Medicine, Baltimore, MD, USA. ${ }^{17}$ Department of Critical Care Medicine, University of Calgary, Calgary, Alberta, Canada. ${ }^{18}$ Department of Surgery, University of Calgary, Calgary, Alberta, Canada. ${ }^{19} \mathrm{EG} 23$ Foothills Medical Centre, Calgary, Alberta T2N 2 T9, Canada.
\end{abstract}

Published online: 17 October 2019

\section{Reference}

1. Doig CJ, Page SA, McKee JL, Moore EE, Abu-Zidan FM, Carroll R, et al. Ethical considerations in conducting surgical research in severe complicated intra-abdominal sepsis. World J Emerg Surg. 2019;14:39. https://doi.org/10.1186/s13017-019-0259-9.

\footnotetext{
* Correspondence: Andrew.kirkpatrick@albertahealthservices.ca

${ }^{17}$ Department of Critical Care Medicine, University of Calgary, Calgary, Alberta, Canada

${ }^{18}$ Department of Surgery, University of Calgary, Calgary, Alberta, Canada

Full list of author information is available at the end of the article
}

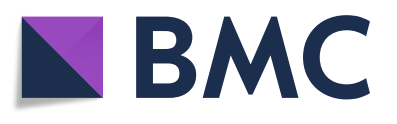

(c) The Author(s). 2019 Open Access This article is distributed under the terms of the Creative Commons Attribution 4.0 International License (http://creativecommons.org/licenses/by/4.0/), which permits unrestricted use, distribution, and reproduction in any medium, provided you give appropriate credit to the original author(s) and the source, provide a link to the Creative Commons license, and indicate if changes were made. The Creative Commons Public Domain Dedication waiver (http://creativecommons.org/publicdomain/zero/1.0/) applies to the data made available in this article, unless otherwise stated. 